This item was submitted to Loughborough's Research Repository by the author.

Items in Figshare are protected by copyright, with all rights reserved, unless otherwise indicated.

\title{
Beam-switching planar parasitic antenna array
}

PLEASE CITE THE PUBLISHED VERSION

http://dx.doi.org/10.1109/LAPC.2014.6996346

PUBLISHER

(c) IEEE

\section{VERSION}

AM (Accepted Manuscript)

\section{PUBLISHER STATEMENT}

This work is made available according to the conditions of the Creative Commons Attribution-NonCommercialNoDerivatives 4.0 International (CC BY-NC-ND 4.0) licence. Full details of this licence are available at: https://creativecommons.org/licenses/by-nc-nd/4.0/

\section{LICENCE}

CC BY-NC-ND 4.0

\section{REPOSITORY RECORD}

Imran, Zohaib, and C.J. Panagamuwa. 2019. "Beam-switching Planar Parasitic Antenna Array". figshare. https://hdl.handle.net/2134/22686. 


\title{
Beam-switching Planar Parasitic Antenna Array
}

\author{
Zohaib Imran and Chinthana Panagamuwa \\ Loughborough University, \\ School of Electronic, Electrical and Systems Engineering, \\ Loughborough, United Kingdom \\ z.imran-10@student.lboro.ac.uk, C.J.Panagamuwa@lboro.ac.uk
}

\begin{abstract}
This paper presents a planar parasitic antenna array that achieves beam switching operation by using the Yagi-Uda principle. The treatment of the Yagi-Uda principle in this paper employs closely-spaced directors as opposed to the traditional inter-director spacing, and this shows advantages in terms of the front-to-back ratio (F/B) and size. The beam switching has been realized in 3 principal beam directions through the selective switching of 4 PIN Diodes on the antenna structure. A fully functional antenna using PIN Diodes with an optimized biasing line arrangement and circuitry has been fabricated, and the results obtained through measurement show good agreement with simulation results. The antenna shows good impedance match and beam-switching characteristics in the Wi-Fi frequency band, opening up the possibility of the antenna's use in practical applications.
\end{abstract}

\section{INTRODUCTION}

With the increased proliferation of wireless communications in recent times, and the increasingly crowded and cluttered RF environment, a host of problems such as multipath fading and interference can occur [1], which have the effect of degrading the communications link and causing inefficient usage of power and network resources. This has necessitated the development of antennas with reconfigurable characteristics, and within this wide domain, "pattern reconfiguration" or "beam-steering" antennas have been a crucial and active area of research, which has seen a lot of iterations and development in recent times, and holds a lot of potential for solving some of the problems encountered in the communications link.

A directive antenna that also has the ability to steer its beam in a number of directions, can allow an operator to point a directive beam pattern towards the intended recipient, and to reduce radiation to or from undesired areas. This has applications in terms of power-saving, security, and reliability. There is a need for achieving this functionality in the cheapest, most practical and miniaturized formats, with the increasing trend towards the realization of once prohibitively expensive and complicated solutions being widely available and used.

The domain of highly directive beam steering antennas has classically seen treatment through the phased array antenna principle, but the high degree of complexity, expense of fabrication, and low efficiency of these types of antennas has precluded their use in some applications. This has seen the development of an alternative approach to beam-steering in the form of parasitic arrays, which exhibit better characteristics in terms of efficiency, cost and complexity. A number of solutions have been developed in this area, which use parasitic elements in conjunction with driven element(s). They control current flow on parasitic elements using switches such as Varactor Diodes [2], RF MEMS [3], PIN Diodes [4-6].

This paper will present the theoretical concept, design, and the performance results of a planar parasitic antenna array, which uses the Yagi-Uda principle and a switching circuit that utilizes PIN Diodes in order to achieve beam steering in three different modes: two directive end-fire beams in opposite directions, and an omnidirectional pattern.

\section{ANTENNA DESIGNA AND THEORY OF OPERATION}

The proposed antenna is a planar Yagi-Uda parasitic array, where a double-sided dipole, which acts as the driven element, is surrounded on both sides by a number of parasitic elements.

The antenna was printed on an $80 \times 80 \mathrm{~mm}^{2}$ substrate with a relative permittivity of 2.365 , loss tangent of 0.0013 and thickness of $1.16 \mathrm{~mm}$. The structure and dimension parameters of the antenna design can be seen in Fig. 1. The dimensions of the antenna are as follows: $L=49.25 \mathrm{~mm}, R L=54.23 \mathrm{~mm}$, $D L=28.32 \mathrm{~mm}, W=4.51 \mathrm{~mm}, C S L=7.06 \mathrm{~mm}, R S=15.7 \mathrm{~mm}$, $I C S=5.9 \mathrm{~mm}, I D S=0.5 \mathrm{~mm}$, and $O L=2.5 \mathrm{~mm}$.

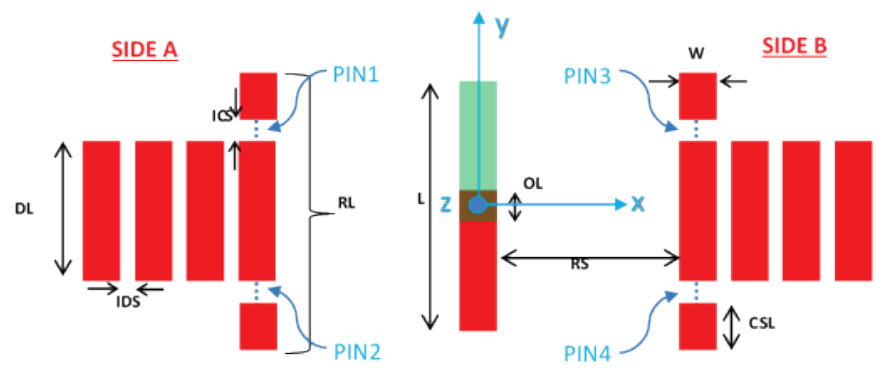

Fig. 1. Antenna design and dimension parameters (Red $=$ Top layer metallization, Green = Bottom layer Metallization) (Not drawn to scale)

Beam switching is achieved by alternatively increasing the electrical lengths of the parasitic elements on sides A and B. With PIN1 and 2 on, the parasitic element on side A acts as a reflector $(R)$ directing the radiation towards side B. With PIN3 and 4 off, the radiation is further augmented due to the presence of 4 directors $(D)$, as per the Yagi-Uda principle [7]. 
This provides a directive beam in the $+\mathrm{X}$ direction, while suppressing the radiated power in the $-\mathrm{X}$ direction. This state is referred to as the $R D$ switching state. By inverting the state of all 4 PIN diodes, the $D R$ switching state is achieved, where radiation is directed towards the $-\mathrm{X}$ direction. In addition to this, a third state $(D D)$ can also be achieved by leaving all the PIN diodes in the off state, which will provide a nearomnidirectional pattern similar to that of an ideal dipole. The simulated 3D Farfield pattern for the 3 switching states can be seen in Fig. 2.

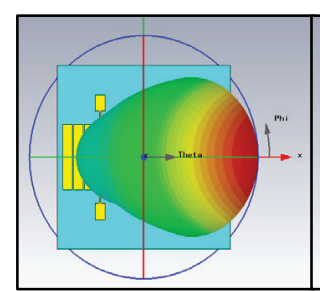

(a)

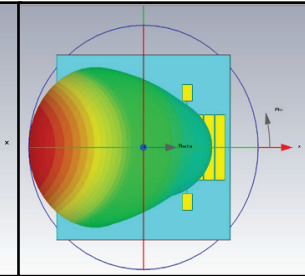

(b)

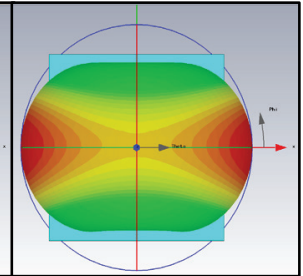

(c)
Fig. 2. Simulated $3 \mathrm{D}$ radiation patterns evaluated at $2.4 \mathrm{GHz}$ for the antenna operating in: (a) RD mode (b) DR mode (c) DD mode

The proposed antenna's radiation mechanism can be explained by the Yagi-Uda principle where the length of the "director" element(s) are slightly shorter, and the "reflector" element(s) are slightly longer with respect to the driven dipole. The shorter length of the "directors" leads to it having a capacitive reactance, and a current that leads the phase of the induced emf. The longer length of the "reflector" results in it having an inductive reactance, and a current that lags the phase of the induced emf [7]. This phase relationship results in the radiation being enhanced in the direction of the "directors", while the radiation towards the "reflector" side is reduced. This theory of operation was confirmed in the simulation domain, through visualizing the surface current densities on the antenna operating in one of its end-fire modes (Fig. 3).

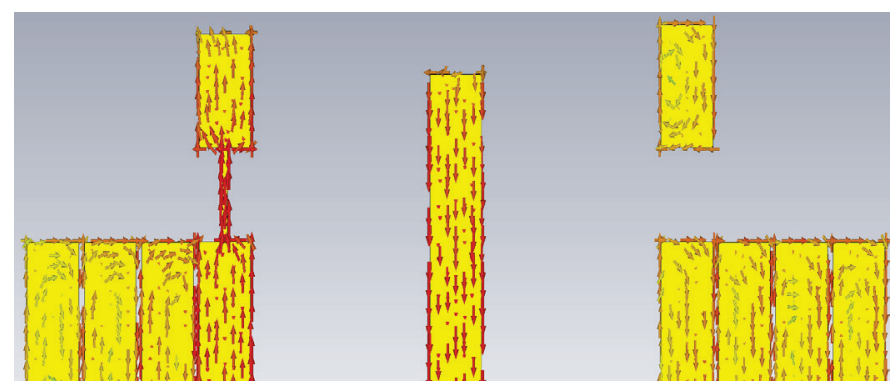

Fig. 3. Surface current distribution of the antenna operating in the RD mode.

The surface currents can be seen to be concentrated mainly on the driven dipole element, and the reflector element. The current on these two elements are in opposite directions which provides the phase relationship wherein the currents on these elements have a destructive interference, causing the radiation emanated from the dipole to be reduced in the direction of the reflector and augmented on the director side.

\section{PARAMETRIC ANALYSIS AND DESIGN}

The antenna has been designed after an extensive series of optimizations achieved via performing parametric sweeps, utilizing the CST Microwave Studio Suite.

The principal and most important parameters that needed to be optimized were those that relate to the characteristics of the main forward beam in one of the switched states (DR and RD). The aim was to achieve a highly directive beam in the end-fire direction, while minimizing the radiated power in the opposite direction. These two properties can be characterized in terms of simulation results as the "Main lobe Magnitude (Gain)" and the "Back Lobe Magnitude (Gain)" respectively, which can then be represented in a single compound result known as the Front-to-Back Ratio (F/B). Therefore the optimization process was primarily focused towards optimizing the different antenna dimensions such as the Director Length/Width, Reflector Length/Width, No. of Directors, Inter-Director Spacing, etc. with respect to the F/B.

Along with the beam characteristics, another task was to make the antenna operational over a functional frequency band, and the band chosen was the Wi-Fi band which spans the frequencies around $2.4 \mathrm{GHz}$, and therefore the optimization procedure focused on behavior at $2.4 \mathrm{GHz}$. As per the antenna's main intended functionality, ensuring that the best possible $\mathrm{F} / \mathrm{B}$ ratio was achieved at $2.4 \mathrm{GHz}$ was a goal that took precedence over making the antenna resonate exactly at $2.4 \mathrm{GHz}$. However, in order to fulfil the functionality for operation in the frequency band, it was important to make sure that the reflection coefficient was better than $-10 \mathrm{~dB}$ at the design frequency, and to this end the parameterization goal was set to achieve a impedance match of at least $-15 \mathrm{~dB}$ at 2.4 $\mathrm{GHz}$, in order to allow for sufficient tolerance of deterioration in performance in the actual fabricated antenna due to fabrication errors, or the deterioration in performance caused by the addition of the surface mount components (SMCs) and the DC biasing lines.

TABLE I. SIMULATED ANTENNA PERFORMANCE WITH VARIATION IN IDS (RD MODE, 2 DIRECTORS)

\begin{tabular}{|c|c|c|c|c|}
\hline $\begin{array}{c}\text { Inter Director } \\
\text { Spacing }\end{array}$ & $\begin{array}{c}\text { Main Lobe } \\
\text { Magnitude } \\
(\mathbf{d B i})\end{array}$ & $\begin{array}{c}\text { Back Lobe } \\
\text { Magnitude } \\
\mathbf{( d B i})\end{array}$ & $\begin{array}{c}\mathbf{3 d B} \\
\text { Angular } \\
\text { width }\end{array}$ & $\begin{array}{c}\text { F/B } \\
(\mathbf{d B})\end{array}$ \\
\hline $30 \mathrm{~mm}(\approx 0.3 \lambda \mathrm{g})$ & 6.82 & -4.88 & $111.7^{\circ}$ & $\mathbf{1 1 . 7}$ \\
\hline $20 \mathrm{~mm}(\approx 0.2 \lambda \mathrm{g})$ & 6.58 & -3.73 & $114.8^{\circ}$ & $\mathbf{1 0 . 3}$ \\
\hline $10 \mathrm{~mm}$ & 6.46 & -4.62 & $119.5^{\circ}$ & $\mathbf{1 1 . 1}$ \\
\hline $0.5 \mathrm{~mm}$ & 6.71 & -8.52 & $120^{\circ}$ & $\mathbf{1 5 . 2}$ \\
\hline
\end{tabular}

The ideal inter-director spacing (IDS) for planar Yagi-Uda arrays for maximum gain in the end-fire direction should be close to $0.2-0.3 \lambda_{g}$ [8-9]. The IDS and the size of the directors were optimized in CST Microwave Studio, with an aim to 
reduce overall size of the antenna, and to achieve the best F/B. Results are presented in Table I. Reducing the IDS to $0.5 \mathrm{~mm}$ $\left(0.005 \lambda_{g}\right)$ causes a $30.2 \%$ increase in the $\mathrm{F} / \mathrm{B}$ ratio, while suffering only a $1.6 \%$ decrease in the main beam gain, compared to when using $0.3 \lambda_{g}$ director spacing.

\section{SWITCHING CIRCUIT AND BIASING LINE DESIGN}

The antenna's switching circuit design involved creating the biasing lines in addition to appropriately sized pads for incorporating the surface-mount components on the antenna, and the copper pads required for soldering the biasing wires from the power source.

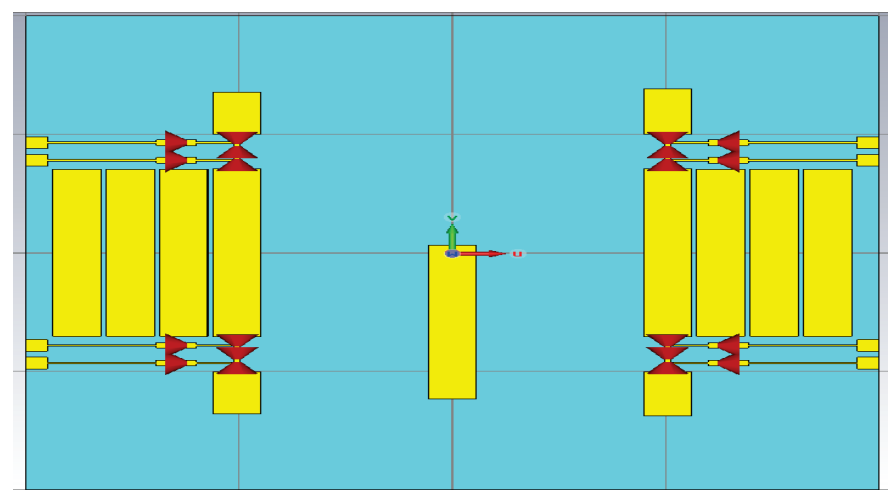

(a)

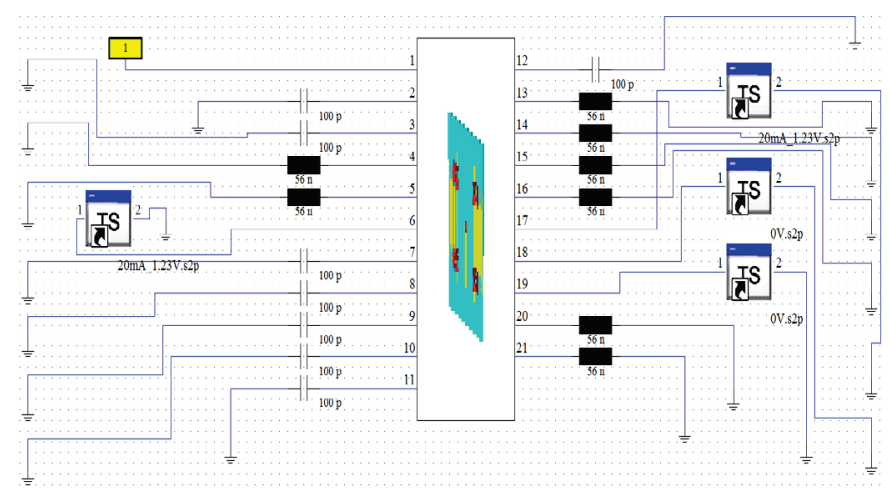

(b)

Fig. 4. CST model for the antenna with biasing lines and components modelled as discrete ports. (b) CST Design Studio Scematic representation

Different designs and arrangements of the biasing lines were trialed with the aim of ensuring that the antenna's radiation characteristics and performance were not disrupted, while ensuring that all the surface mount components can be incorporated without increasing the size of the antenna. The final optimized design can be seen in Fig. 4 (a), alongside the different SMCs on the antenna modelled as discrete ports. Fig. 4 (b) shows how the different discrete ports were defined in the CST Design Studio, with the inductors and capacitors being modelled as lumped elements, and the PIN Diode being modelled by the manufacturer provided Touchstone files.

Fig. 5 shows a closer look at one of the biasing networks that are located on the fabricated antenna's surface, which dictate the switching operation of one of the four PIN Diodes. The biasing lines are $0.3 \mathrm{~mm}$ wide, the minimum width possible to be fabricated using the university's facilities, in order to provide the maximum possible impedance to the RF currents.

The PIN Diodes used for the antenna were the SMP-1345$079 \mathrm{LF}$, and these diodes have a very low capacitance of $0.15 \mathrm{pF}$ in the OFF state, and a very low forward resistance of $1.5 \Omega$ in the ON state.

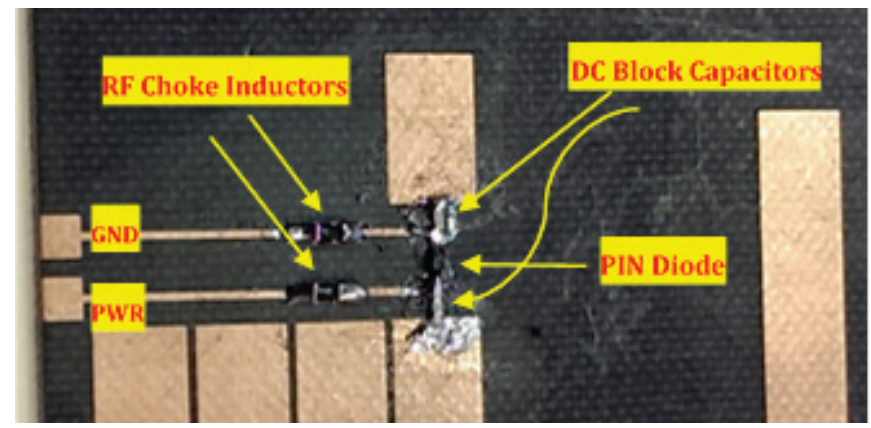

Fig. 5. Biasing lines and circuitry for switching PIN diode

The RF Choke Inductors (Panasonic 667-ELJ-QE56NGFA) have been placed as close as possible to the DC bias lines in order to minimize the dissipation of the RF currents into the bias lines. The choice of RF Choke inductor was driven by the aim of providing the largest possible impedance to the RF currents at $2.4 \mathrm{GHz}$. The $56 \mathrm{nH}$ inductor will provide an impedance of $844.5 \Omega\left(Z_{L}=2 \pi f L\right)$ at $2.4 \mathrm{GHz}$. Another crucial design consideration was the self-resonant frequency of the inductors [10], and these inductors have a SRF of 2500 $\mathrm{MHz}$, which ensures that the behavior of the inductors will still be inductive at $2.4 \mathrm{GHz}$.

The DC blocking capacitors (AVX - 04025A101FAT2A) simplify the biasing arrangement of the PIN Diodes, as the need for a reverse-bias configuration is needed otherwise. The DC block capacitors have been chosen to allow the unimpeded flow of RF currents at $2.4 \mathrm{GHz}$ where an impedance of only $0.663 \Omega\left(Z_{C}=\frac{1}{2 \pi f C}\right)$ will be seen, in addition to serving its main purpose of constraining the DC currents within the required loop in order to avoid the chance of incorrect biasing of the PIN diodes.

The PIN diodes are turned on with a bias current of $30 \mathrm{~mA}$, and in order to apply the appropriate level of biasing current to the system, the apparatus shown in Fig. 6 (b) was used, which includes a Size C battery (1.5V) holder, that has been soldered on to three $100 \Omega$ potentiometers connected to three power ports that can supply a current that is biased according to the potentiometer setting, and a common ground port. Since the apparatus had only 3 ports, the OFF state was simply simulated by leaving the wires coming out of that side of the antenna unattached. This was the simplest solution to implement but it is not a practical solution in real-world terms where it would be desirable to not have to manually detach/reattach the biasing wires to the PIN diodes on one side of the antenna to facilitate Beam-switching. To this end, future work on the antenna will involve the construction of an extra port on the apparatus, with the inclusion of DPST switches to turn off the required PIN diodes. 


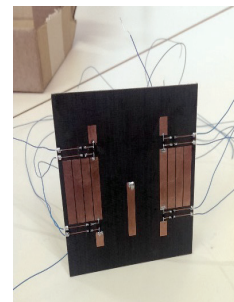

(a)

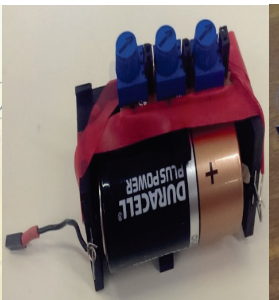

(b)

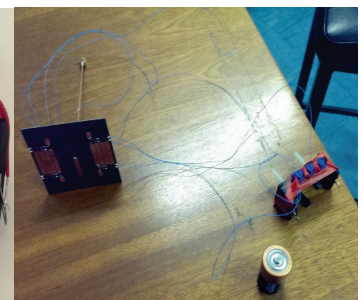

(c)
Fig. 6. (a) Fabricated antenna with the biasing wires soldered on. (b) Biasing current controlling apparatus (c) Full system in conjunction

\section{RESULTS AND ANALYSIS}

The far-field measurements were performed using Loughborough University's Anechoic Chamber facilities (Fig. 7 (a)), and the S11 measurements were performed using the Anritsu 37397D VNA (Fig. 7 (b))

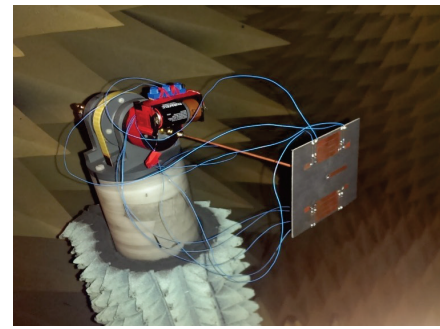

(a)

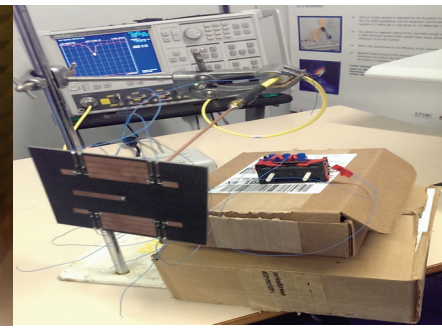

(b)
Fig. 7. (a) Farfield measurment setup (b) S11 measurment setup

Measured and simulated S11 and radiation patterns are shown in Fig. 8 and 9 respectively. Results are summarized in Table II.

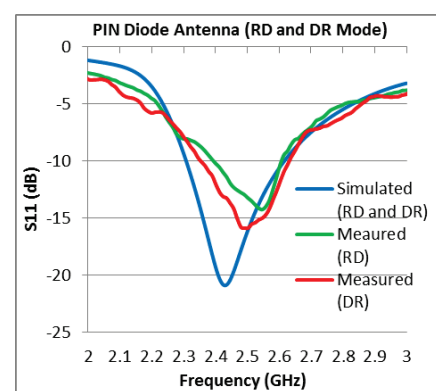

(a)

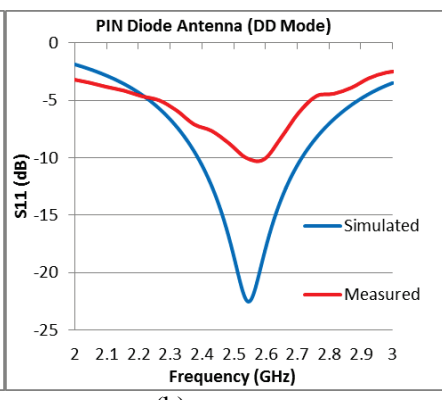

(b)
Fig. 8. Simulated and measured S11 plots for the antenna operating in the: (a) RD and DR modes (b) DD mode

It can be seen in Fig. 9 that the measured radiation patterns show a good agreement with the simulated radiation patterns. The slight discrepancies can be caused due to undesired parasitic radiation and the spurious radiation from the unbalanced coaxial feed. There is also some interference by the antenna positioner which is not optimized for small antenna radiation pattern measurements.

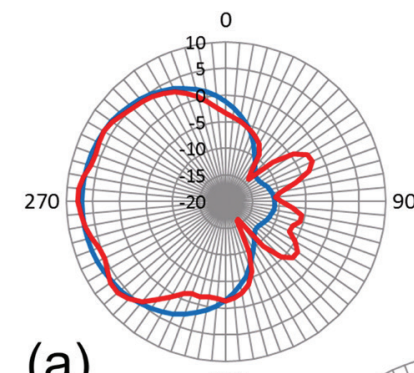

(a)

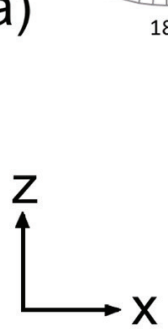

(b)

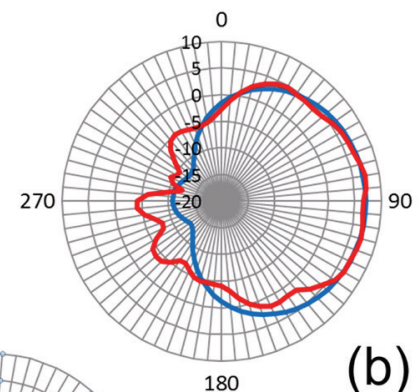

Fig. 9. Simulated (blue lines) and measured (red lines) radation patterns (phi $=0$, theta cuts) for the antenna operating in the: (a) DR mode $(2.385 \mathrm{GHz})$ (b) RD mode $(2.385 \mathrm{GHz})(\mathrm{c}) \mathrm{DD}$ mode $(2.4375 \mathrm{GHz})$

TABLE II. SIMULATED AND MEASURED PERFORMANCE RESULTS FOR THE ANTENNA

\begin{tabular}{|c|c|c||c|c||c|c||}
\hline \multirow{2}{*}{ Mode } & \multicolumn{2}{|c||}{ Gain (dBi) } & \multicolumn{2}{c||}{$\begin{array}{c}\text { Resonant } \\
\text { Frequency (GHz) }\end{array}$} & \multicolumn{2}{c||}{$\begin{array}{c}\text {-10dB Fractional } \\
\text { Bandwidth (\%) }\end{array}$} \\
\cline { 2 - 7 } & Sim. & Meas. & Sim. & Meas. & Sim. & Meas. \\
\hline RD & 7.077 & 6.729 & 2.43 & 2.57 & 11.5 & 9.0 \\
\hline DR & 7.077 & 7.767 & 2.43 & 2.503 & 11.5 & 10.36 \\
\hline DD & 3.024 & 1.45 & 2.55 & 2.595 & 12.5 & 2.14 \\
\hline
\end{tabular}

Fig. 8 shows the measured and simulated S11 responses of the antenna in its three states. The measured S11 shows a reasonable level of agreement with the simulated S11 in the end-fire modes RD and DR. The resonant frequency shifts from $2.43 \mathrm{GHz}$ in simulation to $2.57 \mathrm{GHz}(\mathrm{RD})$ and $2.503 \mathrm{GHz}$ (DR) in measurement, while the impedance match degrades from $-22.8 \mathrm{~dB}$ in simulation to $-15.9 \mathrm{~dB}(\mathrm{RD})$ and $-14.7 \mathrm{~dB}$ (DR) in measurement.

There is a poor agreement between the simulated and measured S11 responses in the omni-directional mode (DD) of the antenna. There seems to a good a good agreement with respect to the resonant frequency, but the measured impedance match $(-10.15 \mathrm{~dB})$ is much pooper in comparison to the simulated impedance match $(-22 \mathrm{~dB})$. This can be attributed to the improper modeling of the PIN diodes within the simulation and due to the simulation not taking the self-resonance frequency (SRF) of the RF choke inductor into account. The resonance in the DD mode occurs around $2.6 \mathrm{GHz}$, which is greater than the SRF $(2.5 \mathrm{GHz})$ of the RF choke inductor used in the antenna, and this can cause undesired behavior by the inductor and therefore compromise its function [10].

The discrepancies in the resonance frequency and the impedance match can be attributed to insufficiently detailed 
modelling of the simulation environment. The simulation model did not take into account the SRF values of the inductors, the effect of the DC bias wires, or the presence of the battery pack in the antenna's near-field. In addition to this the antenna was fed with an ideal "discrete port" in simulation, whereas a more accurate representation could use a model of the $17 \mathrm{~cm}$ RG-402 cable that was used, which would provide an unbalanced feed as was the case with the fabricated antenna, and also model the spurious radiation that can be caused by the coaxial cable. The implementation of these extra details within the simulation will serve as future work on the antenna, and can bring the measured and simulated results closer in agreement to each other. Aside from simulation detailing, discrepancies can also occur due to factors such as the deterioration of the antenna structure being caused due to solder use, fabrication errors, fluctuating arrangement of the biasing wires etc.

The end-fire modes show measured $-10 \mathrm{~dB}$ fractional bandwidths (FBW) of $9 \%$ and $10.36 \%$ respectively which brings them within the generally agreed upon required FBW specification for wireless systems $(7-13 \%)$.

\section{CONCLUSIONS}

This paper presents a beam switching planar parasitic array, which through the selective switching of PIN diodes on the antenna structure, achieves beam steering in three different modes. The design principle was successfully demonstrated as can be seen by the good match achieved between the simulated and measured results.

Through the implementation of closely spaced directors, the antenna has managed to achieve a higher number of director elements in the same form factor, and this helps in the miniaturization of the antenna compared to other designs that utilize traditional director spacing.
In future, the antenna design can be improved by using a more extensively detailed simulation environment, a balanced feed, an RF choke inductor with a higher SRF, and a more constant and reliable arrangement system for holding the biasing wires

\section{REFERENCES}

[1] Theodore S. Rappaport, "Wireless Communications: Principles and Practice (2nd Edition)", Prentice Hall, 2001 (2nd Edition)

[2] Luther, J.J.; Ebadi, S.; Xun Gong, "Electrically-Steerable Parasitic Array Radiator (ESPAR) antenna design for arrays with two and three parasitically-coupled elements," Radio and Wireless Symposium (RWS), 2012 IEEE, vol., no., pp.79,82, 15-18 Jan. 2012

[3] Petit, L.; Dussopt, L.; Laheurte, J.-M., "MEMS-Switched ParasiticAntenna Array for Radiation Pattern Diversity," Antennas and Propagation, IEEE Transactions on , vol.54, no.9, pp.2624,2631, Sept. 2006

[4] Zhang, S.; Huff, G.H.; Feng, J.; Bernhard, J.T., "A pattern reconfigurable microstrip parasitic array," Antennas and Propagation, IEEE Transactions on, vol.52, no.10, pp.2773,2776, Oct. 2004

[5] Jung-Woo Baik, Seongmin Pyo, Tae-Hak Lee, and Young-Sik Kim. "Switchable Printed Yagi-Uda Antenna with Pattern Reconfiguration." ETRI journal 31, no. 3 (2009): 318-320.

[6] Sharma, S.K.; Fideles, F.; Kalikonda, A., "Radiation pattern reconfigurable planar Yagi-Uda antenna," Antennas and Propagation Society International Symposium (APSURSI), 2013 IEEE , vol., no., pp.190,191, 7-13 July 2013

[7] Constantine A. Balanis, "Antenna Theory: analysis and design", John Wiley \& Sons, 1997 (3rd Edition)

[8] Floc'h, J.-M.; Denoual, J.M.; Sallem, K., "Design of printed dipole with reflector and multi directors," Antennas \& Propagation Conference, 2009. LAPC 2009. Loughborough, vol., no., pp.421,424, 16-17 Nov. 2009

[9] Cheng, D.; Chen, C., "Optimum element spacings for Yagi-Uda arrays," Antennas and Propagation, IEEE Transactions on , vol.21, no.5, pp.615,623, Sep 1973

[10] http://www.digikey.co.uk/Web\%20Export/Supplier\%20Content/APIDel evan_197/PDF/API_right_inductor.pdf?redirected $=1$, accessed July $27^{\text {th }}$ 2014 . 